\title{
Etude hydrobiologique de la Vallée d'Ossau (Pyrénées-Atlantiques). I. Répartition et écologie des Ephéméroptères.
}

\author{
G. Vinçon! \\ A.G.B. Thomas ${ }^{2}$
}

Mots clés : Ephemeroptera, écologie, Pyrénées-Atlantiques analyse factorielle des correspondances, classification ascendante hiérarchique, paramètres du milieu. groupements d'espèces.

29 stations lotiques ont été prospectées en Vallée d'Ossau (Pyrénées-Atlantiques) entre 450 et $2150 \mathrm{~m}$ d'altitude et 30 espèces d'Ephéméroptères récoltées. Deux analyses factorielles des correspondances - dont l'une tenant compte des para. mètres du milieu - ainsi qu'une classification ascendante hiérarchique ont été effectuées. Les 5 groupements d'espèces mis en évidence correspondent: I aux ruisseaux très froids de haute altitude, II aux torrents de moyenne montagne et aux ruisseaux de haute altitude à températures estivales élevées, II a aux rivières de piémont, III a aux ruisseaux assez lents et à forte teneur en calcium, III b aux ruisseaux à faible courant et à fond vaseux riche en macrophytes.

Le regime thermique et la vitesse du courant apparaissent nettement conme les principaux facteurs de répartition en montagne. L'altitude, la surface du bassin-versant, le numéro d'ordre du cours d'eau. le régime hydraulique et les caractéristiques physico-chimiques jouent aussi un rôle important. Les préférences écologiques des principales espèces sont discutées.

\section{A hydroblological study of the Ossau Valley (Atlantlc Pyrenees). I. Distribution an ecology of Ephemeroptera.}

Keywords : Ephemeroptera, ecology, Atlantic Pyrences, factorial analyses, ascending hierarchic classification, environmental parameters, groups of species.

29 running-water stations were surveyed in the Ossau valley (Atlantic Pyrenees) between altitudes of $450 \mathrm{~m}$ and $2150 \mathrm{~m}$, and 30 species of Ephemeroptera were recorded. The data were analysed using two factorial analyses, one of which included the environmental parameters, and an ascending hierarchic classification. These analyses divided the species into five groups : I. from very cold streams at high altitude : II. from mountain streams at mean altitude, and streams at high altitude but with high summer temperatures; Ila. from rivers of the piedmont region (lower region of mountains); IIIa. from fairly slow-flowing rivers with a high level of calcium ; IIIb. from very slow-flowing rivers with a muddy bottom rich in macrophytes.

The thermal regime and current speed were the chief factors affecting distribution in the mountains. Altitude, catchment area, stream order, hydrological regime and physical-chemical characteristics also played an important role. The ecological preferences of the principal species are discussed.

La connaissance de la répartition et de l'écologie des Ephéméroptères des Pyrénées est encore très fragmentaire, en particulier sur le versant Nord.

La présente étude faunistique de la vallée d'Ossau a permis de recenser 30 espèces d'Ephéméroptères entre 450 et $2150 \mathrm{~m}$. Ce travail axé sur l'étude de données faunistiques semi-quantitatives, se fixe

1. 15, rue E. Calvat, 38000 Grenoble, France.

2. Laboratoire d'Hydrobiologie, UA 695 du C.N.R.S., Université P. Sabatier, 118, route de Narbonne, 31062 Toulo: sse Cedex, France. pour objet de préciser la distribution spatiale et l'écologie des espèces dominantes du peuplement.

Les seules données antérieures dont nous disposons sur les Ephéméroptères de cette vallée sont celles, sommaires et de déterminations douteuses, de Bertrand et Verrier (1949).

\section{Les stations et leurs paramètres}

Les 29 stations prospectées ( 27 en vallée d'Ossau et 2 dans la haute vallée du Rio Gallego sur le 


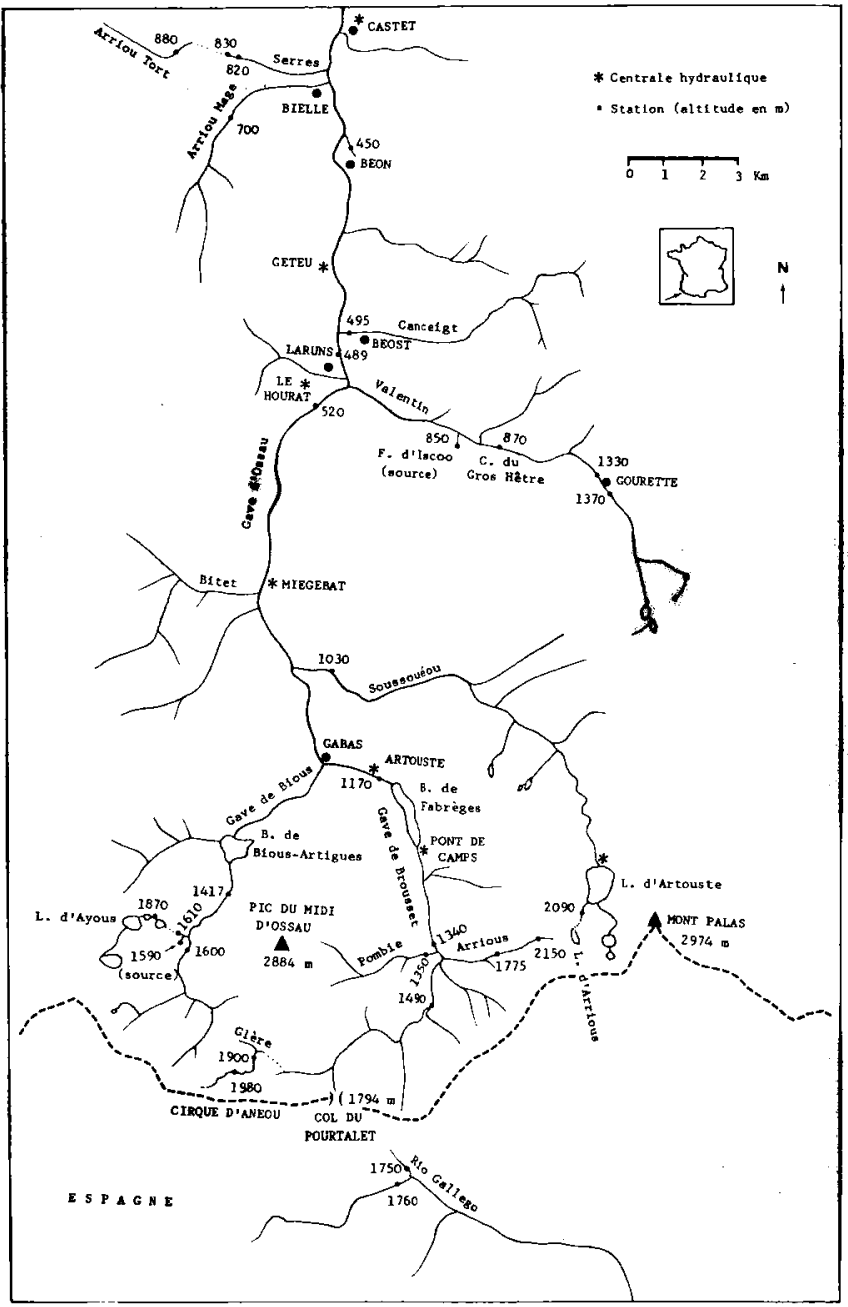

Fig. 1: Réseau hydrographique du Gave d'Ossau. Stations de récoltes et principaux aménagements hydro-electriques. 
Tableau I : Récapitulatif des caractéristiques des stations étudiées dans la vallée d'Ossau et la haute vallé du Rio Gallego. Les codes utilisés sont expliqués dans le tableau $I I$.

\begin{tabular}{|c|c|c|c|c|c|c|c|c|c|}
\hline $\begin{array}{l}\text { Stationn, } \\
\text { alcitude }(m) \text {, } \\
\text { et } n^{*} \text { de code }\end{array}$ & 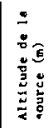 & $\begin{array}{l}0 \\
\dot{E} \\
\dot{x} \\
\dot{E} \\
\dot{E}\end{array}$ & 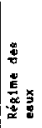 & 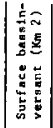 & $\mid \begin{array}{l}0 \\
5 \\
0 \\
0 \\
0 \\
0 \\
0 \\
5 \\
5 \\
2 \\
2\end{array}$ & 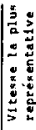 & 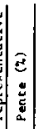 & 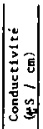 & 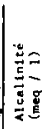 \\
\hline F. $2150(5)$ & 230 & 4,5 & 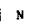 & 0.3 & 1 & M & 30 & 76 & 0.46 \\
\hline D.Ar. $2090(16)$ & 2250 & 5 & & 0,2 & 1 & L & 3 & 76 & 0,54 \\
\hline A.C. 1950 (3) & 2080 & 15 & NT & 1,1 & 1 & $T R$ & 15 & 52 & 0,26 \\
\hline A.G. $1900(4)$ & 2080 & 16 & vT & 1,7 & 1 & $M$ & 5 & 95 & 0,68 \\
\hline D.Ay. 1870 (11) & 2070 & 14 & NT & 1,8 & 2 & $T R$ & 25 & 54 & 0,36 \\
\hline$A r=1775(6)$ & 230 & 9 & 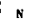 & 2 & 2 & R & 10 & 84 & 0,61 \\
\hline A.R.C. 1760 (2) & 1900 & 15 & NT & 0,8 & 1 & $R$ & 10 & 75 & 0,53 \\
\hline R.G. 1750 (1) & 1800 & 15 & NT & 0,3 & 1 & M & 5 & 272 & 1,19 \\
\hline A.B1. $1610(12)$ & 1650 & 16 & NT & 2 & 1 & TR & 30 & 55 & 0,38 \\
\hline Bi. $1600(13)$ & 2100 & 10 & NT & 12 & 3 & $T R$ & 20 & 124 & 0,98 \\
\hline S.A.B1. 1590 (14) & 1590 & 6,5 & $s$ & 0,2 & 0 & m & 20 & 184 & 1,40 \\
\hline Br. 1490 (7) & 2080 & 14 & NT & 17 & 3 & TR & 10 & 163 & 1,21 \\
\hline B1. 1417 (15 & 2100 & 11 & NT & 18 & 3 & $T R$ & 15 & 107 & 0,88 \\
\hline v. $1370(18)$ & 2280 & 12 & NT & 7 & 3 & TR & 10 & 82 & 0,88 \\
\hline p. 1350 (9) & 2000 & 12 & NT & 6 & 2 & TR & 20 & 73 & 0,50 \\
\hline Br. 1340 (8) & 2230 & 13 & NT & 22 & 3 & R & 5 & 139 & 1,0 \\
\hline v. $1330(19)$ & 80 & 12 & NT & 9 & 3 & TR & 10 & $16 \mathrm{a}$ & 1,30 \\
\hline Br. $1170(10)$ & 2230 & 13,5 & NT & 61 & 3 & $R$ & 6 & 156 & 1,20 \\
\hline 5. $1030(17)$ & 2320 & 13 & $\mathrm{Nr}$ & 52 & 3 & TR & 15 & 90 & 0,60 \\
\hline A.T. 880 (23) & 950 & 20 & $\mathbf{F N}$ & 9 & 1 & b & 1 & 283 & 2,48 \\
\hline v. $870(20)$ & 2280 & 13 & NT & 16 & 3 & $\mathbf{R}$ & 5 & 195 & 1,47 \\
\hline $5.1 .850<21$ & 850 & 8 & 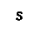 & 0,3 & 0 & R & 30 & 218 & 1,86 \\
\hline Se. $830(24)$ & 950 & 16 & FN & 9 & 1 & 2 & 2 & 402 & 3,68 \\
\hline Se. $820(25)$ & 950 & 17,5 & PN & 9,5 & 1 & M & 10 & 351 & 3,36 \\
\hline A.M. $700(22)$ & 1650 & 12 & NT & 15 & 2 & $T R$ & 15 & 219 & 1,86 \\
\hline $0.520(27)$ & 2320 & 15 & NT & 220 & 4 & R & 3 & 201 & 1,63 \\
\hline c. $495(26)$ & 1650 & 15 & NT & 22 & 3 & 8 & B & 287 & 1,94 \\
\hline $0.489(28)$ & 2320 & 15 & NT & 280 & 4 & $R$ & 1 & 190 & 1,63 \\
\hline R.N.B. 450 (29) & 500 & 9 & $\mathrm{~s}$ & 0,2 & 10 & 2 & 21 & 228 & $1, \%$ \\
\hline
\end{tabular}

versant espagnol : fig. 1) ont été décrites dans un précédent travail (Vinçon 1987). Le tableau I résume leurs principales caractéristiques.

Angelier \& al. (1985) ont montré que cinq paramètres seulement - l'altitude de la station, l'altitude de la source située la plus en amont sur le mème cours d'eau, la pente à la station, la surface du bassin-versant et le régime des eaux - suffisent pour rendre compte de façon fiable de la distribution spatiale des Hydracariens dans les cours d'eau des Pyrénées centrales. A ce complexe stationnel, nous avons rajouté cinq autres paramètres précisant l'environnement à la station: la conductivité, l'alcalinité et la température maximale de l'eau, le numéro d'ordre du cours d'eau et la vitesse du courant.

Ces dix descripteurs du milieu rendent compte, de façon directe ou indirecte, des quatre principaux facteurs de répartition des especes en milieu lotique montagnard : le régime thermique, le débit, la vitesse du courant et la physico-chimie.

Subdivisés en 47 classes (Tableau II), ces dix paramètres ont été traités dans une analyse factorielle des correspondances avec les autres données (stations et espèces).

Voici les abréviations utilisées pour la désignation des stations $(\boldsymbol{r} .=$ ruisseau $; t$. $=$ torrent $;$ riv. $=$ rivière).

Ar. $=\mathbf{r}$. d'Arrious $;$ D.Ar. $=r$. déversoir du lac d'Arrious ; A.G. $=r$. affluent de la Glère $; D . A y .=t$ déversoir du lac d'Ayous : A.R.G. = r. affluent du Rio Gallego ; R.G. $=$ Rio Gallego ; A.Bi. $=r$, affluent de Bious $; \mathrm{Bi}=\mathrm{t}$. de Bious; S.A.Bi. = source affluent de Bious $; \mathbf{B r} .=t$. de Brousset ; V. $=t$. du Valentin $; P$. $=r$. de Pombie $s=t$. du Soussouéou ; A.T. = r. d'Arriou Tort ; S.I. = source d'Iscoo; Se. $=r$. de Serres $; A . M .=r$ d'Arriou Mage $; O .=$ riv. d'Ossau $:$ C. $=\mathrm{t}$. du Canceigt et R.N.B. $=r$. au nord de Béon.

\section{Les espèces}

Sur un total de 30 espèces, 29 ont été recensées dans le réseau du Gave d'Ossau, entre 450 et $2150 \mathrm{~m}$, et 15 dans la haute vallée du Rio Gallego, à 1750 et $1760 \mathrm{~m}$. Ce sont :

Famille des BaEtidaE

genre Baetis Leach, 1815

1. B. alpints Pictet, 1843

= B. carpatica Morton, 1910

$=B$ dovieri Degrange, 1957

2. B. catharus Thomas, 1986

3. B. groupe fuscatus (Linné, 1761)

4. B. gemellas Eaton, 1885

5. B. melanonyx Pictet, 1843

6. B. muticus (Linne, 1758)

$=$ B. pumilus (Bumeister, 1839)

7. B. rhodani Pictet, 1843

8. B. sinaicus Bogoescu, 1931

9. B. vertus Curtis, 1834

10. B. sp. A (groupe latheri Müller-Liebenau, 1967)

genre Centroptilum Eaton, 1869

11. C. Luteolum (Müller, 1776)

12. C. groupe pennulartm Eaton, 1870

Famille des HeptagenIIDaE. genre Epeorus Eaton, 1881

13. E. torrentitum Eaton, 1885

genre Rhithrogena Eaton, 1881

14. Rh. groupe hercyria Landa. 1970 
Tableau II : Classes des paramètres utilisées pour le codage.

\begin{tabular}{|c|c|c|c|c|c|c|c|c|}
\hline \multicolumn{2}{|c|}{$\begin{array}{l}\text { Al ti tude } \\
\text { (m) }\end{array}$} & \multicolumn{3}{|c|}{$\begin{array}{l}\text { Al ti tude de la } \\
\text { source }(\mathrm{m})\end{array}$} & $\mathrm{T}_{(0}^{\circ} \max ^{\circ}$ & \multicolumn{2}{|c|}{$\begin{array}{c}\text { Régime des } \\
\text { eaux }\end{array}$} & $\begin{array}{c}\text { Bassin-versant } \\
(\mathrm{km} 2)\end{array}$ \\
\hline & $<700$ & \multirow{2}{*}{\multicolumn{3}{|c|}{$\begin{array}{ll}\text { Aso source } & \begin{array}{l}\text { elle même } \\
\text { ell }\end{array}\end{array}$}} & $\mathrm{Tl}<5$ & RO & source (S) & Bl $0-1$ \\
\hline & $700-1100$ & & & & $\pi 25-8$ & R1 & nival (N) & B2 $1-4$ \\
\hline A3 & $1100-1500$ & \multirow{2}{*}{\multicolumn{3}{|c|}{$\begin{array}{l}\text { Asl }<1500 \\
\text { As2 } 1500-1900\end{array}$}} & T3 $8-11$ & R2 & nival de & B3 $4-16$ \\
\hline & $1500-1900$ & & & & T4 $11-14$ & & transition & B4 $16-64$ \\
\hline & $>1900$ & \multirow{2}{*}{\multicolumn{3}{|c|}{$\begin{array}{l}\text { As } 3 \quad 1900-2200 \\
\text { As } 4>2200\end{array}$}} & T5 $14-17$ & $\mathrm{~B}_{3}$ & 1] & $B 5>64$ \\
\hline & & & & & $\mathrm{T} 6>17$ & & nival (PN) & \\
\hline \multicolumn{2}{|c|}{$\begin{array}{l}\text { Numéro } \\
\text { d'ordre }\end{array}$} & \multicolumn{3}{|c|}{$\begin{array}{l}\text { Vitesse du } \\
\text { courant }\end{array}$} & $\begin{array}{l}\text { Pente } \\
(\%)\end{array}$ & \multicolumn{2}{|c|}{$\begin{array}{l}\text { Conductivité } \\
(\mu \mathrm{S} / \mathrm{cm})\end{array}$} & $\begin{array}{l}\text { Alcalinité } \\
(\text { meq } / \text { l) }\end{array}$ \\
\hline No & crénal & v1 & \multicolumn{2}{|c|}{ lente ( $L$ ) } & $\mathrm{Fl}<2$ & $\mathrm{Cl}$ & $<70$ & $A c l<0,5$ \\
\hline N1 & 1 & v2 & \multicolumn{2}{|c|}{ moyenne (M) } & P2 $2-5$ & C2 & $70-140$ & AC2 $0,5-1$ \\
\hline N2 & 2 & v3 & \multicolumn{2}{|c|}{ rapide (R) } & P3 $5-10$ & $\mathrm{C}_{3}$ & $140-280$ & Ac3 $1-2$ \\
\hline N3 & 3 & \multirow[t]{2}{*}{ V4 } & \multirow{2}{*}{\multicolumn{2}{|c|}{ très rapide (TR) }} & P4 $10-20$ & \multirow{2}{*}{\multicolumn{2}{|c|}{$>280$}} & \multirow[t]{2}{*}{ Ac4 $2-4$} \\
\hline N4 & 4 & & & & P5 $>20$ & & & \\
\hline
\end{tabular}

15. Rh. kimminsi Thomas, 1970

16. $R h$. loyolaea Navás, 1922

17. $R$ h. semicolorata (Curtis, 1834), sensu Sowa (1970)

18. Rh. strenua Thomas, 1982

genre Ecdyonurus Eaton, 1868

19. E. angelieri Thomas, 1968

20. E. forcipula (Pictet, 1843), sensu Thomas (1968 a)

21. E. venosus (Fabricius, 1775), sensu Thomas (1968 a)

genre Electrogena Zurwerra et Tomka, 1985

22. E. groupe lateralis (Curtis, 1834)

Famille des EPHEMERELLIDAE

genre Ephemerella Walsh, 1862

23. E. ignita (Poda, 1861)

24. E. major Klapálek, 1905

Famille des CaEnidae

genre Caenis Stephens, 1835

25. C. beskidensis Sowa, 1973

Famille des LePTOPHLEBIIDAE

genre Paraleptophlebia Lestage, 1917

26. P. submarginata (Stephens, 1835)

genre Habrophlebia Eaton, 1881

27. H. lauta Eaton, 1884

genre Habroleptoides Schcenemund. 1928

28. $H$. berthelemyi (Thomas, 1968)

29. H. confusa Sartori et Jacob, 1986

$=H$. modesta sensu auct.

Famille des EPHEMERIDAE

genre Ephemera Linné, 1746

30. E. danica Müller, 1764
A ces 30 espèces 1 , nous avons ajouté deux autres taxons représentés à l'état larvaire : Ecdyonurus sp. et Epeorus sp. En effet, les larves des espèces pyrénéennes appartenant à ces deux genres ne sont pas identifiables de façon très satisfaisante dans l'état actuel de nos connaissances.

Lorsqu'elles sont mentionnées, les déterminations spécifiques (Ecdyonurus angelieri, Ecd. forcipula, $E c d$. venosus et Epeorus torrentium) ont été réali. sées à partir des imagos capturées, ce, dans quelques stations seulement ; aussi la connaissance de leur répartition dans le réseau du Gave d'Ossau estelle encore assez fragmentaire.

Répartition et abondance des espèces sont indiquées dans le tableau III.

\section{Les groupements d'Ephéméroptères et les relations espèces-paramètres des milieux}

Pour décrire la structure du peuplement en Ephéméroptères de la vallée d'Ossau, nous avons procédé

1. Sur les 30 especes de cette liste, 5 au moins n'ont pu étre nomurnées avec certitude et feront l'objet de travaux taxonomiques ultérieurs. 
Tableau III : Répartition des Ephéméroptères dans les vallées d'Ossau et du haut Rio Gallego. Les codes des espèces sont ceux utilisés dans les deux A.F.C. (fig. 2, 3, 4 et 5). Les totaux ne tiennent pas compte des taxons génériques Epeorus et Ecdyonurus lorsque des espèces de ces genres ont été identifiees (imagos) dans la station.

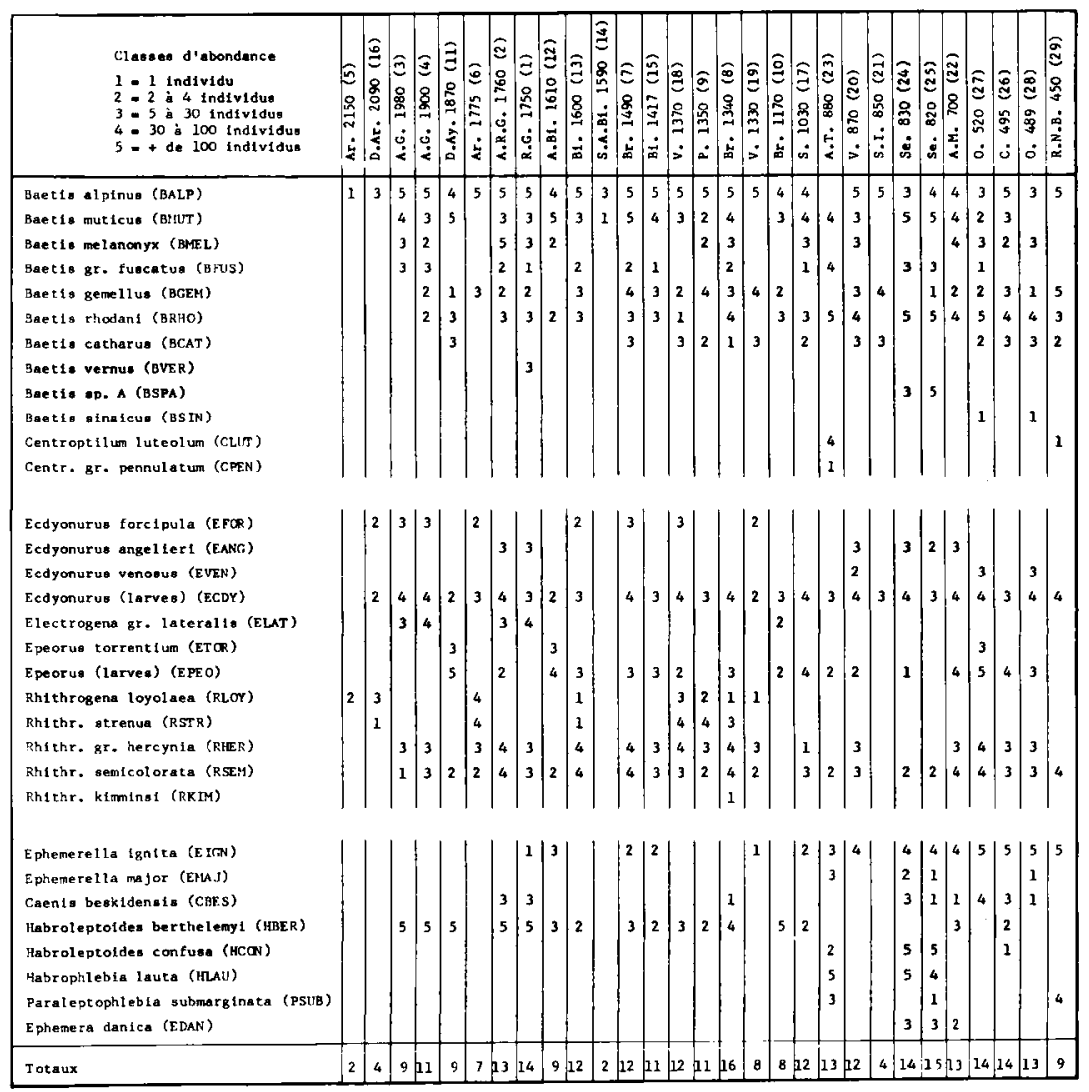


à deux analyses factorielles des correspondances (A.F.C.).

La première a traité globalement trois ensembles de données : les 29 stations, les 32 taxons et les dix paramètres subdivisés en 47 classes.

Pour chaque station, l'abondance des espèces dans les prélèvements a été cotée selon cinq niveaux (correspondant aux récoltes cumulées):

$1=1$ seul individu : espèce très rare

$2 \simeq 2$ à 4 individus : espèce rare

$3=5$ à 30 individus : espèce assez abondante

$4=30$ à 100 individus : espèce abondante

$5=$ plus de 100 individus : espèce très abondante.

La deuxièmé analyse a porté seulement sur deux ensembles de données: les 29 stations et les 32 taxons.

\subsection{Analyse factorielle des correspondances: paramètres-stations-espèces}

Les quatre premiers axes rendent compte de $52 \%$ de l'inertie totale du nuage des points.

Signification des principaux axes

Dans la figure 2, les différentes valeurs des principaux paramètres sont reliées pour faciliter l'interprétation des axes. Les espèces et les stations ne sont pas figurées pour alléger la réprésentation graphique; elles le sont sur la figure 3.

- L'axe I (19\% de l'inertie totale) paraît essentiellement déterminé par la température de l'eau et, à un moindre degré, par un ensemble de paramètres qui varient régulièrement de l'amont vers l'aval : la vitesse du courant, le régime des eaux, l'altitude de la source.

Sur l'axe II (13\%) se projettent dans un ordre croissant : les numéros d'ordre des cours d'eau et aussi - avec une très faible inversion entre $\mathrm{B} 2$ et $\mathrm{B} 3$ les surfaces des bassins-versants.

- L'axe III (11,5\%) n'a pas été représenté car il apporte peu de renseignements par rapport à la seconde A.F.C., avec surtout un fort isolement du crénal.

Il est intéressant de noter qu'aucun des axes I à VI ne rend compte de façon satisfaisante de l'altitude en elle-même.

\subsection{Analyse factorielle des correspondances:} stations-espèces

Les quatre premiers axes rendent compte de $61 \%$ de l'inertie totale du nuage des points : $24,5 \%$ pour le premier et respectivement 16,11 et $10 \%$ pour les trois suivants.

Plusieurs groupes de stations apparaissent assez bien délimités sur les deux représentations graphiques (plans des axes I et II : fig. 4 et I et III : fig. 5). Ils représentent différents types de biotopes.

\section{a) Groupes de stations}

Les groupes les mieux individualisés sont indépendants des paramètres mesurés par l'opérateur (non inclus dans cette analyse); ils n'en sont donc que plus fiables pour traduire l'affinité des espèces visà-vis des milieux qu'elles colonisent.

Pour chaque groupe seront précisés successivement : les stations qu'il rassemble, les systèmes d'axes sur lesquels il apparait le mieux et les caractéristiques du biotope qu'il représente.

\section{Groupe 1}

- stations 5, 16 et 6 ;

- bien individualisé sur les plans des axes I-II et IIII (fig. 4 et 5 );

- ruisseaux froids à très froids $\left(\mathrm{T}^{\circ} \max\right.$ de 5 à $\left.9^{\circ} \mathrm{C}\right)$, en haute altitude $(>1700 \mathrm{~m})$.

Leurs sources sont situées au-dessus de $2200 \mathrm{~m}$ et leur régime des eaux est de type nival. Ce biotope correspond au " sommerkalten Bach " des auteurs allemands (Brehm \& Ruttner 1926).

Groupe 2

- stations 13,18, 9 et 19 ;

- bien délimité sur le plan des axes I-II (fig. 4) où il est séparé du groupe 3 par le groupe 5 :

- torrents froids $\left(\mathrm{T}^{\circ} \max \leqslant 12^{\circ} \mathrm{C}\right.$ ), en moyenne montagne $(1300.1600 \mathrm{~m})$. Ils sont issus de sources situées en haute altitude ( 2000 à plus de $2200 \mathrm{~m}$ ) et ont un régime des eaux de type nival de transition.

\section{Groupe 3}

Moins homogène, il rassemble un plus grand nombre de stations (16) et peut être scindé en trois sousensembles $(a, b$ et $c)$ contigus sur le plan des axes I-Il (fig. 4), mais nettement séparés le long de l'axe III (fig. 5). 


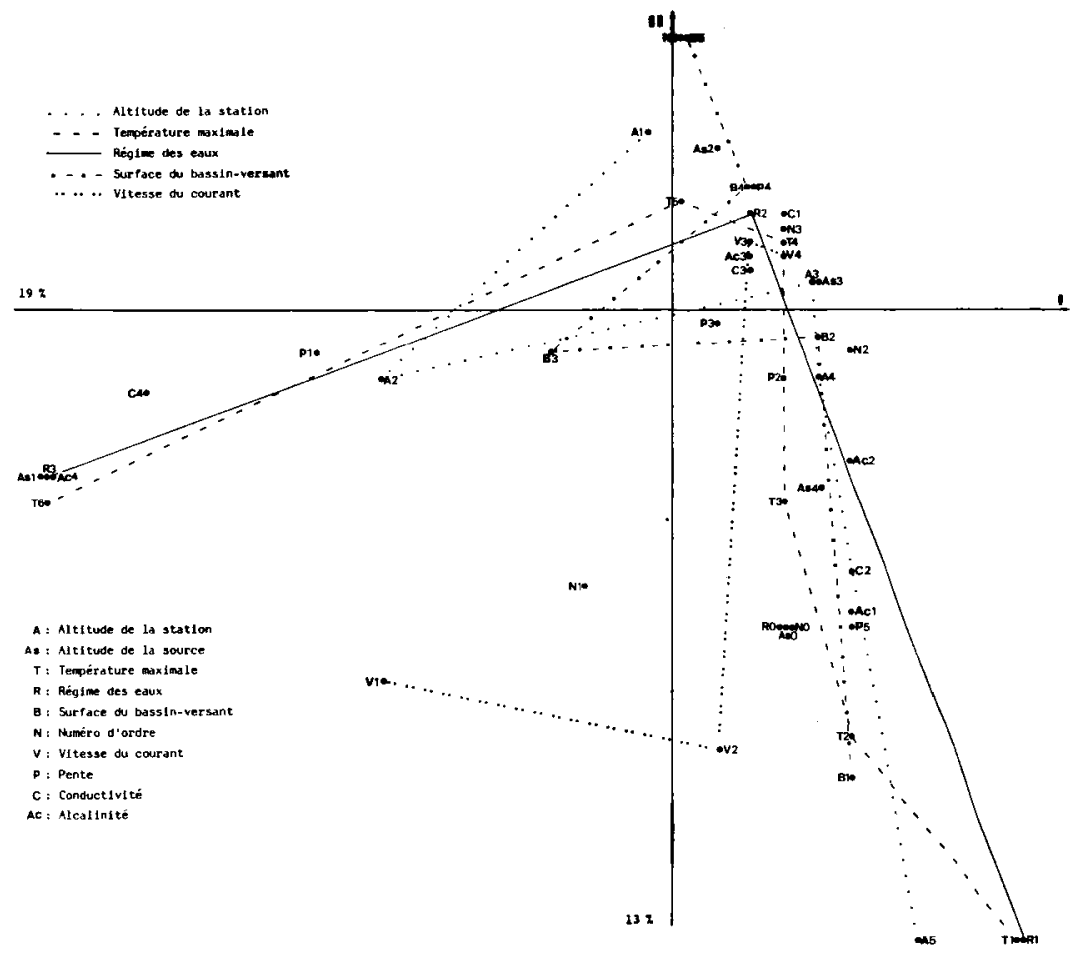

Fig. 2 : Première analyse factorielle des correspondances (A.F.C.) paramètres-stationsespèces. 1' partie :évolution de quelques paramètres importants dans le plan des axes I et II (les espèces et les stations sont représentées sur la fig. 3. Pour le codage, voir le tableau (I).

Sous-groupe $3 a$

- stations 1, 2, 3 et 4 ;

- ruisseaux de haute altitude ( $>1700 \mathrm{~m})$ à températures estivales plutôt élevées, atteignant facilement $15-16^{\circ} \mathrm{C}$. Leurs sources sont situées entre $1800 \mathrm{et}$ $2100 \mathrm{~m}$ et leur régime des eaux est de type nival de transition. Ce biotope correspond au a sommerwarmen Bach " (Brehm \& Ruttner op. cit.).
Sous-groupe $3 b$

- stations $11,12,7,15,8,10,17,20,22$ et 26 ;

- torrents de moyenne montagne (jusqu'à $1500 \mathrm{~m}$ ) et déversoirs de lacs de haute altitude (stations 11 et 12); températures estivales moyennes à assez élevées $\left(11\right.$ à $16^{\circ} \mathrm{C}$, mais le plus souvent 13 à $\left.16^{\circ} \mathrm{C}\right)$ et régime nival de transition. 


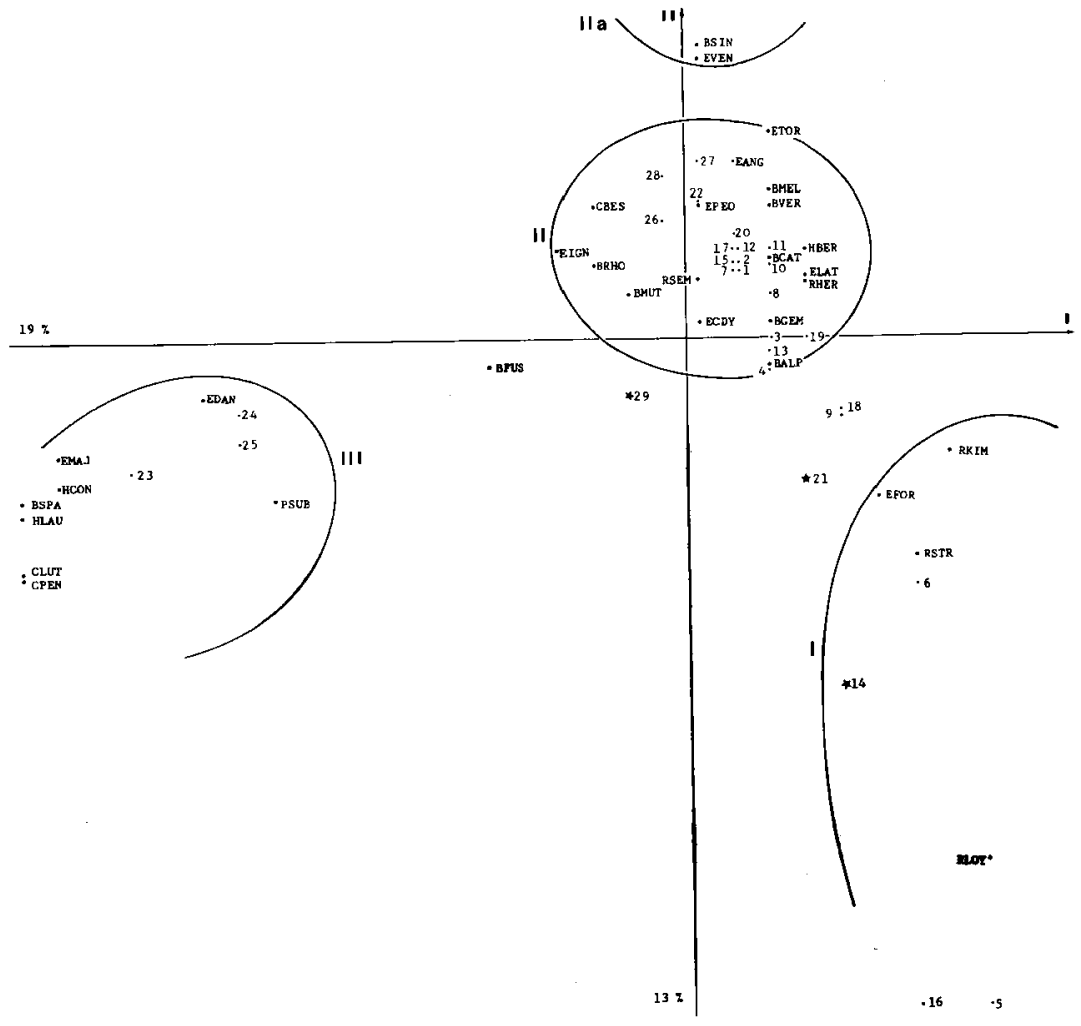

Fig. 3 : Première A.F.C. : paramètres-stations-espèces. $2^{2}$ partie : groupements d'espèces dans le plan des axes I et II (les valeurs des paramètres sont représentées sur la fig. 2. Pour le codage des stations, voir le tableau I et pour celui des espèces, le tableau III). 


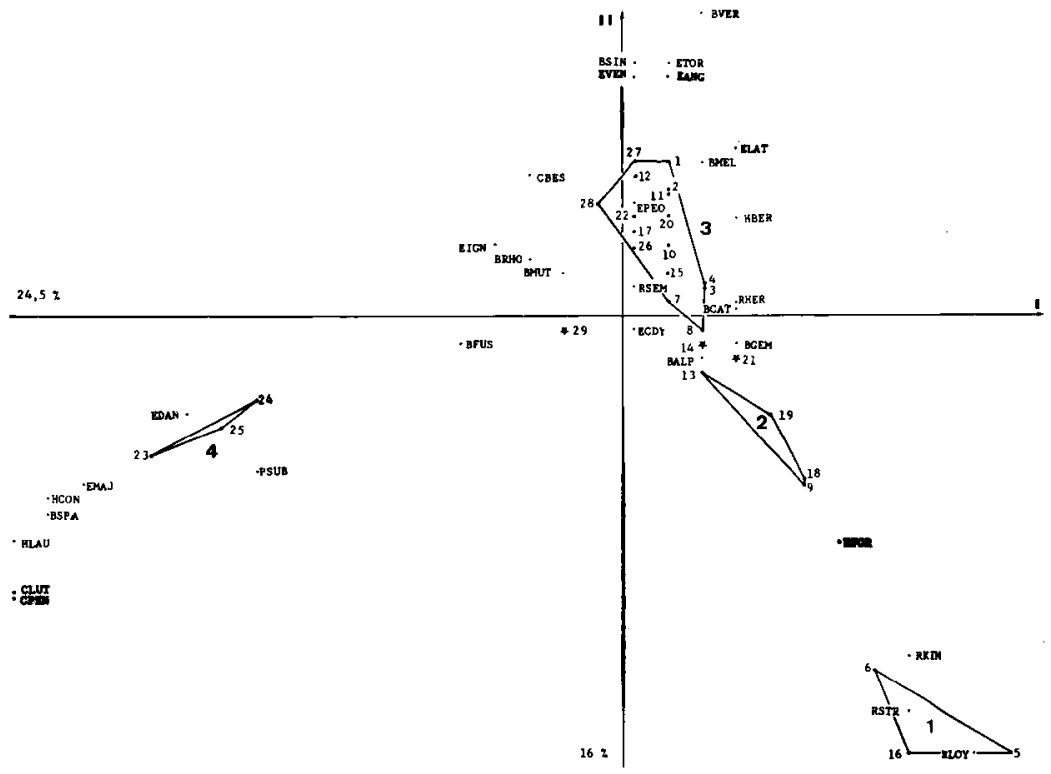

Fig. 4 : Deuxième A.F.C. : stations-espèces. Plan des axes I et II : les groupes de stations.

Sous-groupe $3 \mathrm{c}$

- stations 27 et 28 ;

- rivières de piémont $(500 \mathrm{~m})$, bassin-versant et débit importants, régime nival de transition.

\section{Groupe 4}

- stations 23, 24 et 25 ;

- bien individualisé sur les plans des axes I-II et IIII (fig. 4 et 5) :

- ruisseaux de moyenne montagne $(800-900 \mathrm{~m})$ à températures estivales assez élevées pour l'altitude (16 à $20^{\circ} \mathrm{C}$ ) et régime pluvio-nival.

Sur les plans des axes I-II (fig. 5) et I-IV (ce dernier non représenté), ce groupe se subdivise en deux sous-ensembles :
Sous-groupe $4 a$

- stations 24 et 25, milieu calcaire (encroûtant).

Sous-groupe $4 b$

- station 23, milieu très lent à fond vaseux, riche en macrophytes aquatiques.

Groupe 5 (crénal)

- stations 14,21 et 29 ;

- intercalé sur le plan des axes I-II (fig. 4) entre les groupes 2 et 3 . Il apparaît aussi nettement individualisé sur le plan des axes I-III de l'analyse paramètres-stations-espèces (non représenté).

Dans les diverses figures, les trois stations du crénal sont signalées par une étoile.

Ces différents groupes et leurs subdivisions présentent un net gradient thermique croissant du $\mathbf{1}^{\text {e }}$ 


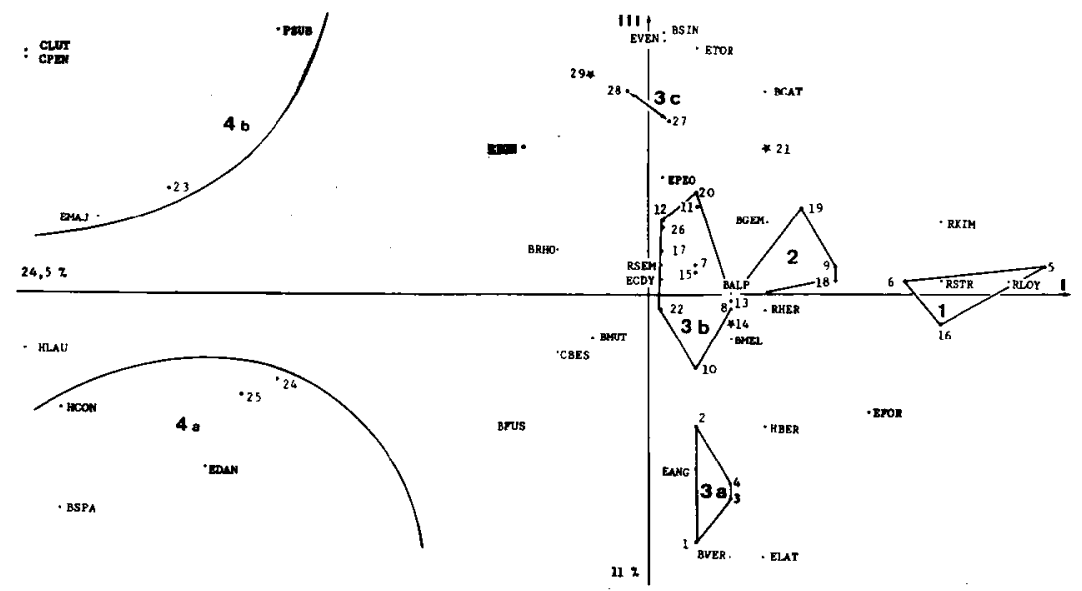

Fig. 5 : Deuxième A.F.C. stations-espèces. Plan des axes I et III : les groupes de stations.

au $4^{e} ;$ le $5^{e}$ (crénal) se distingue par des températures stables et basses toute l'année.

\section{b) Signification des principaux axes}

- Comme dans l'analyse paramètres-stationsespèces, l'axe I (24,5\% de l'inertie totale) est déterminé essentiellement par le régime thermique du cours d'eau selon un gradient de température croissant de la droite vers la gauche.

La vitesse du courant contribue également à la définition de cet axe : les faciès les plus lents sont situés à gauche et les plus rapides à droite.

- L'axe II (16\%) paraît représenter un facteur de spécialisation. Il permet d'opposer des stations de montagne aux caractéristiques moyennes (températures modérées, régime nival de transition) situées audessus du point d'intersection des axes, à des stations aux caractéristiques extrêmes : milieux froids de haute altitude à régime nival (groupe 1) et ruisseaux de moyenne montagne à températures estivales élevées et à régime pluvio-nival (groupe 4).

Il oppose ainsi des espèces sténotopes (sténothermes d'eau froide proches du groupe 1, ou thermophiles, proches du groupe 4) à des espèces plus eurytopes rassemblées au-dessus du point d'intersection des axes.

Remarquons que les trois stations du crénal (aux paramètres écologiques particulièrement stables) se projettent sur cet axe près de l'origine, de même que le taxon générique Ecdyonurus sp., apparaissant fort peu typé car plurispécifique et par conséquent à large valence écologique.

- L'axe III (11 \%) traduit essentiellement le facteur débit, avec la succession : ruisseaux de haute altitude à pente faible à modérée ( $3 \mathrm{a}$ ), torrents de moyenne montagne ( $3 \mathrm{~b}$ ) et rivières de piémont (3c).

- Sur le plan des axes I-IV (non représenté), les sousgroupes $4 \mathrm{a}$ et $4 \mathrm{~b}$ sont encore plus individualisés que sur l'axe III.

\subsection{Les groupements d'espèces déduits des deux A.F.C.}

Sur les figures 3 à 5, et en particulier la fig. 3, peuvent être reconnus plusieurs groupements d'espèces correspondant à des biotopes différents. Sur 
cette dernière figure, les espèces eurytopes se trouvent regroupées près du point d'intersection des axes. Pour définir chaque groupement, nous ferons donc une distinction entre les espèces a fondamentales "d'un biotope et les especes " accessoires", plus eurytopes, pouvant appartenir aussi à d'autres groupements.

a) Groupement I sténotherme d'eau froide à Rhithrogena loyolaea

\section{Espèces fondamentales}

Rhithrogena loyolaea

Rhithrogena strenua

\section{Espèces accessoires}

Rhithrogena kimminsi Ecdyonurus forcipula Baetis alpinus
Ce groupement d'especes correspond au groupe de stations $n^{\circ} 1$ de la $2^{*}$ A.F.C. (stations-espèces), relatif aux ruisseaux froids de haute altitude.

Il est caractérisé par les espèces sténothermes d'eau froide du genre $R$ hithrogena $(R h$. loyolaea et Rh. strenua).

$R h$. kimminsi, proche de ce groupement, en particulier sur le plan I-II (fig. 4), a néanmoins été considérée comme accessoire par sécurité, compte tenu de sa relative rareté.

Ecdyonurus forcipula vit dans quelques cours d'eau de haute altitude avec des températures estivales assez élevées (stations 3 et 4). Sur l'axe I elle apparait ainsi plus eurytherme que $R h$. loyolaea et $R h$. strenua. De plus, dans l'analyse stations-especes, elle est située en position intermédiaire entre les groupes 1 et 2 ( $\mathrm{fig} .4$ ) et les groupes 1 et 3 ( $\mathrm{fig} .5$ ), aussi l'avons-nous considérée seulement comme accessoire.

Baetis alpinus, bien que constante dans ce groupement, n'en est pas caractéristique à cause de sa grande valence écologique. En effet, si Sowa (1975) et Thomas (1975) ont montré que les deux espèces les plus alticoles, en Pologne et sur le versant Nord des Pyrénées, sont $B$. alpinus et $\boldsymbol{R} h$. loyolaea, la première peut être très abondante en basse altitude : Sukop (1973) signale d'importantes populations de B. alpinus dans un cours d'eau karstique de Moravie à 315 et $265 \mathrm{~m}$ d'altitude. On peut seulement affirmer que l'espèce présente une fourchet te thermique préférentielle relativement basse $\left(5\right.$ à $13^{\circ} \mathrm{C}$ selon Belfiore 1983).

$R h$. loyolaea est au contraire beaucoup plus spécialisée (Tableau IV). Les observations d'Humpesch \& Elliott (1980) en donnent une explication : ces auteurs ont montré que les valeurs maximales du pourcentage d'éclosion des cufs étaient obtenues entre 1,9 et $5,2^{\circ} \mathrm{C}$ seulement, avec peu d'éclosions au-delà de $10^{\circ} \mathrm{C}$.

Le groupement I est lié sur les axes I-II $($ fig. 2 et 3) aux paramètres : altitude supérieure à $1500 \mathrm{~m}$ (A4 et A5), températures estivales inférieures à $10^{\circ} \mathrm{C}$ mais le plus souvent net tement moins ( $T 1, T 2$ et T3), sources supérieures à $2200 \mathrm{~m}$ (As4), régime nival à nival de transition ( $R 1$ et $R 2$ ) et forte pente (P5).

Remarque: pour l'instant, compte tenu du petit nombre de stations étudiées, nous sommes incapables de caractériser les torrents froids de moyenne altitude de la vallée d'Ossau (groupe de stations $n^{\circ} 2$ ) par un groupement d'espèces exclusif. Ces cours d'eau abritent en effet à la fois les especes sténothermes du groupement $i$ (en particulier Rh. kimminsi) et des espèces plus eurythermes mais préférant des eaux assez froides (B. gemellus, Rh. gr. hercynia).

Souvent eurytopes et moins diversifiés que les espèces d'Hydracariens, de Trichoptères ou de Plé. coptères, les Ephéméroptères représentent sans doute un ordre moins favorable que les précédents pour la description des communautés typiques d'habitats assez voisins.

b) Groupement II intermédiaire à Habroleptoides berthelemyi

Espèces fondamentales Espèces accessoires

Baetis catharus

Baetis gemellus

Baetis melanonyx

Rhithrogena gr. hercynia

Habroleptoides berthelemyi

Baetis alpinus
Baetis muticus
Baetis hodani
Epeorus torrentium
Rhithrogena semicolorata
Ecdyonurus angelieri
Electrogena gr. lateralis (3a)
Ephemerella ignita (3b)

Il correspond aux groupes de stations $3 \mathrm{a}$ et $3 \mathrm{~b}$ (fig. 4), c'est-à-dire aux ruisseaux de haute montagne à températures estivales relativement élevées et aux torrents de moyenne montagne.

Ce groupement reste encore mal défini surtout pour deux raisons : certaines espèces, identifiées seulement sur imagos (Ecd. angelieri, Ep. torrentium ${ }^{1}$ ), sont situées de façon relativement peu précise sur les différentes représentations : en effet, les chasses d'Ephémères sont toujours très aléatoires et, en conséquence, les listes de stations

1. Ep. sylvicola n'a pas été identifiée (sur adultes or) mais sa pro sence en vallee d'Ossau reste possible. 
Tableau IV : Préférences écologiques des Ephéméroptères recensés dans les vallées d'Ossau et du haut Rio Gallego (vitesse du courant $: T R=$ très rapide, $R=$ rapide, $M=$ moyenne, $L=$ lente. $+=$ espèce fréquente ou abondante, $\bullet=$ espèce rare. " nombre d'individus moven dans les stations où l'espèce est présente; ${ }^{\star \star}$ non compris les sources ca relles repré. sentent tom milieu particulien.

\begin{tabular}{|c|c|c|c|c|c|c|c|c|c|}
\hline & 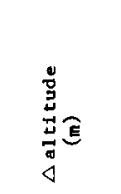 & 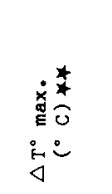 & 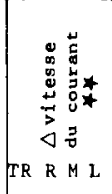 & 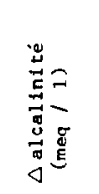 & 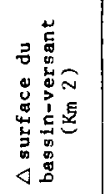 & 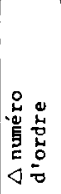 & 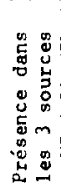 & 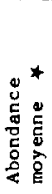 & 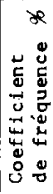 \\
\hline B. alpinus & $450-2150$ & $4,5-17,5$ & +++ & $0,3-3,7$ & $0,2-280$ & $0-4$ & 3 & 118 & 97 \\
\hline B. muticus & $500-2000$ & $10-20$ & ++++ & $0,3-3,7$ & $0,2-280$ & 0.4 & 1 & 62 & 76 \\
\hline B. melanonyx & $500-2000$ & $12-16$ & +++ & $0,3-1,9$ & $0,3-280$ & $1-4$ & 0 & 18 & 45 \\
\hline B. gr. fuscatus & $500-2000$ & $10-20$ & ++++ & $0,3-3,7$ & $0,3-220$ & $1-4$ & 0 & 10 & 45 \\
\hline B. gemel1us & $450-1900$ & $9-16$ & +++ & $0,4-3,4$ & $0,2-280$ & $0-4$ & 2 & 17 & 72 \\
\hline B. rhodant & $450-1900$ & $10-20$ & ++++ & $0,4-3,7$ & $0,2-280$ & 0.4 & $I$ & 40 & 72 \\
\hline B. catharus & $450-1900$ & $12-15$ & ++ & $0,4-2$ & $0,2-280$ & 0.4 & 2 & 7 & 45 \\
\hline $\mathrm{B}$. vernus & 1750 & 15 & - & 1,2 & 0,3 & 1 & 0 & 9 & 3 \\
\hline B. $\mathrm{sp} \cdot \mathrm{A}$ & $800-850$ & $16-17,5$ & ++ & $3,4-3,7$ & $9-9,5$ & 1 & 0 & 61 & 7 \\
\hline B. sinaicus & 500 & 15 & - & 1,6 & $220-280$ & 4 & 0 & 1 & 7 \\
\hline C. luteolum & $450-900$ & 20 & & $2-2,5$ & $0,2-9$ & $0-1$ & 1 & 21 & 7 \\
\hline C. gr. pennulatum & 880 & 20 & - & 2,5 & 9 & 1 & 0 & 1 & 3 \\
\hline Ec. forcipula & $1300-2100$ & $5-16$ & +++ & $0,3-1,3$ & $0,2-17$ & $1-3$ & 0 & & \\
\hline Ec. angelieri & $700-1750$ & $12-17,5$ & $+++\cdot$ & $0,5-3,7$ & $0,3-16$ & $1-3$ & 0 & & \\
\hline Ec. venosus & $500-900$ & $13-15$ & - & $1,5-1,6$ & $16-280$ & $3-4$ & 0 & & \\
\hline El.gr. lateralis & $1150-2000$ & $13,5-16$ & - ++ & $0,3-1,2$ & $0,3-61$ & $1-3$ & 0 & 26 & 17 \\
\hline Ep. torrentium & $500-1900$ & $14-16$ & ++ & $0,4-1,6$ & $1,8-220$ & $1-4$ & 0 & & \\
\hline Rh. loyolaea & $1350-2150$ & $4,5-13$ & $++\ldots$ & $0,5-1,3$ & $0,2-22$ & $1-3$ & 0 & 11 & 28 \\
\hline Rh. strenua & $1350-2100$ & $5-13$ & ++ & $0,5-1$ & $0,2-22$ & $1-3$ & 0 & 31 & 21 \\
\hline $\mathrm{Rh} \cdot \mathrm{gr}$. hercynia & $500-2000$ & $9-16$ & +++ & $0,3-1,9$ & $0,3-280$ & $1-4$ & 0 & 24 & 62 \\
\hline Rh. semicolorata & $450-2000$ & $9-20$ & $+++\cdot$ & $0,3-3,7$ & $0,2-280$ & 0.4 & 1 & 19 & 83 \\
\hline Rh. kimansi & 1350 & 13 & - & 1 & 22 & 3 & 0 & 1 & 3 \\
\hline E. Ignita & $450-1750$ & $12-20$ & +++ & $0,4-3,7$ & $0,2-280$ & $0-4$ & I & 46 & 52 \\
\hline E. major & $500-900$ & $15-20$ & $\cdots+$ & $1,6-3,7$ & $9-280$ & $1-4$ & 0 & 4 & 14 \\
\hline Cn. beskidensis & $500-1750$ & $12-17,5$ &.+++ & $0,5-3,7$ & $0,3-280$ & $1-4$ & 0 & 12 & 31 \\
\hline Ha. ber thel emy i & $500-2000$ & $10-16$ & +++ & $0,3-1,9$ & $0,3-61$ & $1-3$ & 0 & 58 & 55 \\
\hline Ha. confusa & $500-900$ & $15-20$ & ++ & $1,9-3,7$ & $9-22$ & $1-3$ & 0 & 94 & 14 \\
\hline Habroph. Iauta & $800-900$ & $16-20$ & ++ & $2,5-3,7$ & $9-9,5$ & 1 & 0 & 101 & 10 \\
\hline P. submarginata & $450-900$ & $17,5-20$ & $\cdot+$ & $2-3,4$ & $0,2-9,5$ & $0-1$ & 1 & $3 i$ & 10 \\
\hline Eph. danica & $700-850$ & $12-17,5$ & ++ & $1,9-3,7$ & $9-15$ & $1-2$ & 0 & 8 & 10 \\
\hline
\end{tabular}


incomplètes pour les genres Ecdyonurus et Epeorus. En outre, la présence d'espèces eurytopes (B. alpi$n u s, R h$. semicolorata...) à proximité du point d'intersection des axes rend plus difficile la distinction entre espèces fondamentales et accessoires.

En fait, ce peuplement est de type intermédiaire, avec des espèces pour la plupart à large valence écologique.

Le groupement II est lié (fig. 2 et 3 ) aux paramètres : altitude inférieure à $1500 \mathrm{~m}$ (Al et $\mathrm{A} 3$ ), températures estivales comprises entre 11 et $17^{\circ} \mathrm{C}$ (T4 et T5), sources situées entre 1500 et $2200 \mathrm{~m}$ (As 2 et As3), régime nival de transition (R2), pente de 5 à $20 \%(\mathrm{P} 3$ et $\mathrm{P} 4)$ et vitesse du courant moyenne à très rapide (V2, V3 et V4).

c) Sous-groupe IIa de piémont à Ecdyonturus venosus

\section{Espèces fondamentales}

Baetis sinaicus Ecdyonurus venosus
Espèces accessoires

Baetis alpinus
Baetis catharus
Baetis melanonyx
Baetis rhodani
Epeorus torrentitum
Rhithrogena gr, hercynia
Rhithrogena semicolorata
Ecdyonurus angelteri
Ephemerella ignita
Caenis beskidensis

Un groupement d'espèces - que nous avons désigné Ila - paraît être le prolongement du précédent en basse altitude (groupe de stations $3 c:$ fig. 5). En raison du petit nombre de nos stations situées en piémont, nous préférons le considérer pour l'instant comme une subdivision du précédent. Il possède d'ailleurs plusieurs espèces en commun avec le groupement II, tandis qu' $H$. berthelemyi disparaît.

$E c d$. venosus est difficile à séparer de ce que nous considérons encore comme son espèce jumelle : Ecl. forcipula (voir Thomas 1968a). Nous pensons toujours que les deux formes sont distinctes, avec remontée en haute altitude de la seule forcipula (jusqu'à $2300 \mathrm{~m}$ dans les Pyrénées centrales : Thomas 1970). Ecd. venosus serait beaucoup moins alticole et cantonnée au piémont, tout comme $E c d$. dispar (non trouvée en vallee d'Ossau). A l'appui de ce concept, citons les travaux de Macan (1957 et 1962) selon lesquels $E c d$. venostis apparaît dans les grandes rivières à fond pierreux alors qu'elle est absente en amont.
B. sinaicus est, selon Belfiore (1983), une composante typique du rhithron et vit sur substrat pierreux. En fait, il s'agit certainement d'une espèce de piémont pouvant s'accommoder de forts débits. Ainsi, Kamler (1965) la cite des grands cours d'eau de piémont entre 500 et $650 \mathrm{~m}$, dans les Beskides, et Sowa (1975) des rivières moyennes et grandes des Carpates. Selon Krno(1978), elle n'excède pas $800 \mathrm{~m}$ en Tchécoslovaquie.

Le sous-groupement II a, situé en position extrême sur l'axe II ( $f$ ig. 2), est en particulier lié aux paramètres : altitude inférieure à $700 \mathrm{~m}$ (A1), températures estivales comprises entre 14 et $17^{\circ} \mathrm{C}$ (T5), régime nival de transition ( $R 2$ ), surface du bassinversant comprise entre 70 et $280 \mathrm{~km}^{2}$ (B5) et numero d'ordre du cours d'eau égal à 4 (N4).

\section{d) Groupement III thermophile à Habrophlebia lauta}

Nettement séparé des autres ( $f i g .3$ et 4 ), il colonise les ruisseaux de moyenne montagne à températures estivales élevées et pente modérée à très faible (groupe de stations 4).

Il se subdivise en deux sous-ensembles le long des axes III ( $f i g .5$ ) et IV (non représenté).

Sous-groupement IIIa à Habroleptoides confusa
Espèces fondamentales

Baetis sp. A

Habroleptoides confusa

Habrophlebia latsa

Ephemera danica

\section{Espèces accessoires}

\section{Baetis alpinus}

Baetis gr. fuscatus

Baetis muticus

Baetis rhodani

Rhithrogena semicolorata

Ecdyonurus angelieri

Ephemerella ignita

Ephemerella major

Caenis beskidensis
Il caractérise les ruisseaux de la vallée d'Ossau à températures estivales relativement élevées et à fortes teneurs en calcium (sous-groupe de stations 4a).

Il rassemble trois espèces qui se développent en grande abondance dans le ruisseau encroûtant de Serres à 820 et $830 \mathrm{~m}$ et qui sont très peu fréquentes dans le reste du réseau hydrographique étudié : Baetis sp. A (gr. lutheri), Habroleptoides confusa et Habrophlebia lauta.

$H$. lauta et E. danica ont déjà été signalées dans des cours d'eau calcaires (Illies 1952, Guillouzic 1965, Thomas 1970). 
Le sous-groupement IIla est lié aux caractéristiques : altitude entre 700 et $1100 \mathrm{~m}$ (A2); températures supérieures à $17^{\circ} \mathrm{C}$ (T6), régime pluvio-nival (R3), vitesse lente (V1), conductivité supérieure à $280 \mu \mathrm{s} / \mathrm{cm}(\mathrm{C} 4)$ et alcalinité comprise entre 2 et 4 meq/l (Ac4).

Sous-groupement IIIb à Paraleptophlebia submarginata

\section{Espèces fondamentales}

Centroptilum luteolum

Centroptilum gr. pennulatum

Ephemerella major

Habrophlebia lauta

Paraleptophlebia submarginata

\section{Espèces accessoires}

Baetis gr. fuscatus

Baetis muticus

Baetis rhodani

Ephemerella ignita

Il caractérise les faciès à courant lent, à fond envasé et riche en macrophytes aquatiques (sousgroupe de stations $4 b$ ).

Il rassemble des espèces lénitophiles et phytophiles: Centroptilum lateolum et $C$. gr. pennulatum (Macan 1979. Maitland 1980); Paraleptophlebia submarginata (Macan 1952, Belfiore 1983), ainsi que des espèces beaucoup plus eurytopes comme $E$. ignita ou B. rhodani.

H. lauta, située entre les groupes de stations $4 a$ et $4 \mathrm{~b}$ (fig. 5) peut être considérée comme caractéristique des deux sous-groupements IIIa et IIIb à la fois : elle se développe en abondance dans les deux biotopes correspondants et n'a pas été rencontrée ailleurs en vallée d'Ossau

Le sous-groupement IIIb est lié (fig. 2 et 3 ) aux paramètres : altitude entre 700 et $1100 \mathrm{~m}$ (A2), températures estivales supérieures à $17^{\circ} \mathrm{C}(\mathrm{T} 6)$, régime pluvio-nival (R3), pente inférieure à $2 \%(\mathrm{P} 1)$ et faible vitesse du courant (V1).

\section{Conclusion}

Les cinq groupements et sous-groupements d'espèces que nous venons de définir correspondent à cinq types de biotopes montagnards: ruisseaux froids à très froids de haute montagne (I), torrents de moyenne montagne (II), rivières de piémont (IIa), ruisseaux lents et calcaires encroûtants (IIIa) et ruisseaux de moyenne altitude à fond envasé et riche on macrophytes aquatiques (IIIb).

Il existe toutefois deux cas particuliers importants :

- les torrents froids de moyenne montagne abritent, eux, une riche communauté mixte composée d'une part, d'espèces sténothermes d'eau froide appartenant au groupement $I$ et d'autre part, d'espèces plus eurythermes du groupement II :

- quant aux ruisseaux de haute altitude à températures estivales élevées, ils renfermeńt en majorité des espèces du groupement II, c'està-dire des formes qui vivent généralement à plus basse altitude. Décamps (1967) a remarqué un phénomène analogue en haute vallée d'Aure. Ainsi, certaines espèces de Trichoptères qui vivent habituellement dans les cours d'eau de basse ou moyenne altitude se développent-elles au-dessus de $2000 \mathrm{~m}$ dans des vallons bien abrités et exposés à un fort ensoleillement : Estibère, Port Bielh, Aumar...

Les limites du groupement II restent ainsi, de loin, les moins bien définies. Nous espérons pouvoir les préciser ultérieurement sur d'autres vallées des Pyrénées, avec un nombre de stations bien supérieur.

Enfin, soulignons qu'il n'existe pas, dans nos récoltes, d'Ephéméroptères crénobiontes. Tout au plus, quelques espèces peuvent-elles se montrer crénophiles d'eau froide : $B$. alpinus, $B$. calharus et $B$. gemellus - et seule la première tolère des températures très basses tout au long de l'année.

\subsection{Classification ascendante hiêrarchique des espèces}

Cette classification des Ephéméroptères (fig. 6), réalisée sur le tableau des données de la $2^{e}$ A.F.C. (logiciel ADDAD), permet de connaittre à quel niveau sont reliées les espèces fréquemment associées. Elle permet aussi de traduire l'isolement de certains groupements d'espèces qui se rattachent aux autres à des niveaux de liaison très faibles.

Voici les principaux ensembles qui apparaissent :

Le premier nceud sépare très nettement les peuplements torrenticoles (sténothermes d'eau froide ou plus eurythermes), des peuplements d'eau calme, thermophiles. L'inertie de ce nœud est de $18 \%$ du total.

\section{a) Peuplement sténotherme d'eau froide et rhéophile de haute montagne}

Ce premier ensemble est nettement individualisé puisqu'il se rattache à l'ensemble le plus proche à un niveau de liaison inférieur à $40 \%$; l'inertie du 


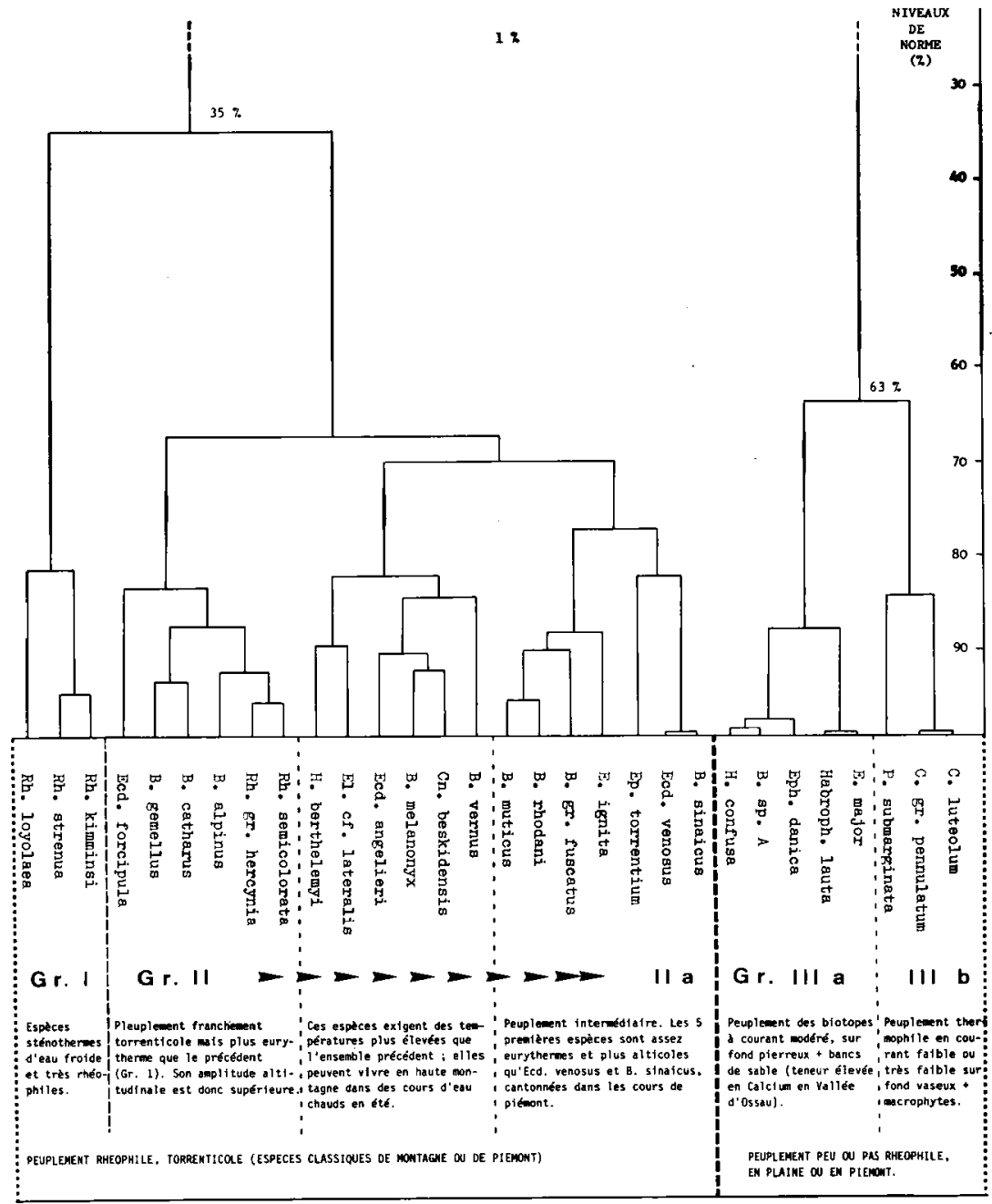

Fig. 6 : Classification ascendante hiérarchique des espèces sur le tableau des données de la $2^{c}$ A.F.C. 
noud est de $12,4 \%$. Il correspond au groupement I à Rhithrogena loyolaea (fig 3 à 5). Ecdyonurus forcipula n'y apparaît pas, sans doute parce qu'il s'agit d'une espece alticole un peu plus eurytherme (Tableau IV).

\section{b) Peuplement rhéophile des torrents de moyenne montagne et des rivières de piémont}

Ce second ensemble, relativement isolé puisque rattaché au précédent à un niveau de liaison inférieur à $40 \%$, rassemble les espèces des groupements II et Ila de l'A.F.C. (fig. 3), caractéristiques des torrents de moyenne montagne et des rivières de piémont.

Il se subdivise lui-même en trois principaux sousensembles (\% d'inertie des nouds compris ent re 6.7 et 5,8 ) qui se raccordent à des niveaux de liaison supérieurs à $60 \%$ :

- le premier comprend des espèces alticoles dont le développement peut s'accommoder de températures maximales relativement basses: Ecd. forcipula, Baetis gemellus, B. catharus, B. alpinus, $R$ hithrogena gr. hercynia et $R h$. semicolorata. Ces espèces rhéophiles sont cependant moins sténothermes que les trois Rhith rogena de l'ensemble précédent;

- les deux sous-ensembles suivants regroupent des espèces encore plus thermophiles. Certaines peuvent se montrer franchement alticoles lorsque les températures estivales sont élevées (en particulier Habroleptoides berthelemyi et Electrogena gr. lateralis), mais la plupart sont surtout fréquentes et abondantes en moyenne altitude. A l'extrême, $E c d$. venosus et $B$. sinaicus, fortement corrélées, caractérisent plus particulièrement les rivières de piémont (groupement IIa).

c) Peuplement thermophile et lénitophile de piémont-moyenne montagne

Ce dernier ensemble, qui correspond au groupement III de l'A.F.C. (fig. 3), se rattache aux autres à un niveau de liaison très faible (1 \%), ce qui souligne son isolement. Il est lui-même scindé en deux sous-ensembles ; le premier (IIIa) : milieu encroûtant en courant modéré et le second (IIIb) : faciès lent à très lent avec fond vaseux.

Conclusion

Cette hiérarchisation confirme les résultats des deux A.C.F.
Les principaux groupements d'espèces (I, II-I Ia et IIIa-IIIb) apparaissent nettement isolés ; ce sont les paramètres : température, altitude et vitesse du courant qui expliquent le mieux leur isolement. Ces trois groupements correspondent également à trois types de régime des eaux : groupement $\mathbf{I}$ : régime nival à nival de transition, groupement II-IIa : régime nival de transition, groupement IIIa-IIIb: régime pluvio-nival.

Les autres paramètres (surface du bassin-versant, numéro d'ordre du cours d'eau, alcalinité et conductivité) expliquent, quant à eux, les subdivisions à l'intérieur de ces principaux ensembles:

- la surface du bassin-versant et le numéro d'ordre du cours d'eat, principalement corrélés à l'altitude (voir la première A.F.C., fig. 2), ont permis de scinder assez distinctement le groupement II-IIa en torrents de moyenne montagne (II) et rivières de piémont (Ila) :

- les données physico-chimiques (alcalinité et conductivité), lorsqu'elles prennent des valeurs très élevées pour des eaux de montagne, comme c'est le cas dans le ruisseau de Serres à 820 et $830 \mathrm{~m}$, contribuent aussi à déterminer un peuplement particulier (sous-groupe IIIa).

\section{Préférences écologiques des espèces}

A l'appui des représentations graphiques, les tableaux IV et $\mathrm{V}$ permettent quelques commentaires sur l'écologie des principales espèces.

\section{Genre Baetis}

B. alpinus confirme sa très large valence écologique. Elle peut vivre dans des torrents, issus de névés, dont la température ne dépasse jamais $3-4^{\circ} \mathrm{C}$ en été (plus de $2600 \mathrm{~m}$ d'altitude : Thomas 1975). Dans ces conditions extrêmes, seule présente, elle surpasse Rhithrogena loyolaea et est l'espèce d'Ephéméroptères la plus cryophile de la faune française. Sa tolérance vis-à-vis de la conductivité est, elle aussi, considérable pour une espèce montagnarde, puisque nous avons enregistré au minimum $31 \mu \mathrm{s} / \mathrm{cm}$ à $2560 \mathrm{~m}$, en vallée d'Aure, et au maximum $402 \mu \mathrm{s} / \mathrm{cm}$ à $830 \mathrm{~m}$, en vallée d'Ossau. Dans nos relevés, elle est à la fois l'espèce la plus abondante et la plus fréquente.

B. gemellus est une espèce assez peu connue. Nous l'avons récoltée avec une fréquence équivalente à celle de son espèce jumelle, $B$. rhodani, mais en 
Tableau V: Classement des principales especes (ccefficient de fréquence supérieur à $20 \%$ et aisément identifiables sur larves), selon la moyenne des températures maximales atteintes par les cours d'eau (colonne de gauche). Altitudes moyennes correspondantes à droite. $\sigma=$ écart type $; \bar{m}=$ moyenne.

\begin{tabular}{lrrrrr} 
& $\begin{array}{r}n \\
\text { st. }\end{array}$ & $\begin{array}{c}\mathrm{T}^{\circ} \mathrm{C} \\
(\overline{\mathrm{m}})\end{array}$ & \multicolumn{1}{c}{$\sigma$} & $\begin{array}{c}\text { alt. } \\
(\overline{\mathrm{m}})\end{array}$ & $\sigma$ \\
\hline Rh. loyolaea & 7 & 9,4 & 3,4 & 1668 & 347 \\
Rh. strenua & 6 & 10,2 & 2,9 & 1588 & 301 \\
B. alpinus & 28 & 12,4 & 3,4 & 1307 & 523 \\
B. catharus & 13 & 12,7 & 2,2 & 1035 & 461 \\
B. gemellus & 21 & 12,9 & 2,5 & 1206 & 496 \\
Rh. gr. hercynia & 18 & 13,2 & 2,0 & 1287 & 494 \\
H. berthelemyi & 16 & 13,5 & 1,8 & 1427 & 420 \\
Rh. semicolorata & 24 & 13,7 & 2,6 & 1234 & 503 \\
B. muticus & 22 & 13,8 & 2,8 & 1288 & 458 \\
B. rhodani & 21 & 14,0 & 2,5 & 1160 & 491 \\
B. melanonyx & 13 & 14,2 & 1,4 & 1215 & 561 \\
E. ignita & 15 & 14,2 & 2,7 & 979 & 438 \\
B. gr. fuscatus & 13 & 14,7 & 2,6 & 1332 & 472 \\
C. beskidensis & 9 & 14,8 & 1,6 & 967 & 518
\end{tabular}

moindre abondance. $B$. gemellus est nettement plus sténotherme d'eau froide que $B$. rhodani comme on peut le voir en projection sur l'axe I des deux A.F.C. ; ces deux espèces présentent toutefois 17 stations en commun $(68 \%)$.

B. rhodani, très répandue, a semble-t-il des exigences thermiques assez voisines de celles de $B$. mut $i$. cus, seconde espèce en vallée d'Ossau par la fréquence et l'abondance. Elles colonisent toutes deux 19 stations communes $(79 \%)$ et peuvent éventuellement se développer en haute altitude, mais seulement dans des biotopes chauds en été, tout comme Habroleptoides berthelemyi.

B. catharus, espèce récemment décrite (1986), est assez fréquente ( $45 \%$ des stations) mais relativement peu abondante. Sa répartition en vallée d'Ossau (13 stations) est recouverte à $100 \%$ par celle de B. alpinus. Il en est de même dans d'autres vallèes et c'est sans doute la raison pour laquelle cette espèce (paracerque court et coloration banale) a été confondue si longtemps avec B. alpinus. Elle vit surtout en moyenne altitude dans des eaux fraîches sous couvert d'arbres feuillus.

Voici, par ordre décroissant, les pourcentages de stations qu'elle possède en commun avec les principales espèces de Baetis (à coeff. de fréquence> $20 \%$ ) :

- gemellus: 54,5

- alpinus : 46,4

- rhodani : 41,7

- melanony $x: 38,9$

- muticus : 37.5

-gr. fuscalus: 18,2

L'affinité la plus grande est vis-à-vis de $B$. gemellus, et l'on constate que les projections des deux espèces sur l'axe I des deux A.F.C. sont pratiquement superposées.

B. melanonyx, dont l'écologie est assez mal connue, apparait elle aussi plus thermophile et occupe une position intermédiaire parmi les espèces du genre Baetis. Mais cela devra être confirmé.

\section{Genre Epeorus}

Les présentes observations confirment qu'E. tor. rentium (terra typica: les Pyrénées françaises) est beaucoup plus fréquente - et sans doute plus abondante-qu'E. sylvicola dans les Pyrénées et les Prépyrénées (Berthélemy \& Thomas 1967).

E. torrentium a été rencontrée entre 520 et $1870 \mathrm{~m}$. En haute altitude, elle est relativement rare sauf dans deux déversoirs de lacs à courant très rapide et à température relativement élevée: le déversoir du lac d'Ayous à $1870 \mathrm{~m}$ et l'affluent du torrent de Bious à $1610 \mathrm{~m}$.

\section{Genre Rhithrogena}

Les tableaux IV et V montrent, eux aussi, que $R h$. loyolaea et $R h$. strenua sont bien les deux espèces d'Ephéméroptères les plus strictement alticoles du réseau hydrographique étudié.

\section{Genre Ephemerella}

E. ignita est très fréquente et abondante dans les cours d'eau de piémont $(450-1000 \mathrm{~m})$. Elle est beaucoup plus rare en haute montagne mais peut tout de même se développer en faible abondance dans des. déversoirs de lacs comme l'affluent de Bious à $1610 \mathrm{~m}$ ou des ruisseaux chauds en été comme le Rio Gallego à $1750 \mathrm{~m}$. 
E. major, nettement moins abondante et moins fréquente, présente une affinité pour les milieux les plus chauds (groupenent IIIb), où elle cohabite avec E. ignita, plus eurytherme ; nous ne l'avons jamais rencontrée au-dessus de $900 \mathrm{~m}$.

\section{Genre Caenis}

Notre citation de $C$. beskidensis, décrite des Carpates polonaises $(300.600 \mathrm{~m})$ par Sowa (1973), est la première pour les faunes de France et d'Espagne (Thomas, Prévot et Vinçon 1986). Cette espèce a été rencontrée assez fréquemment dans les cours d'eau de piémont entre 450 et $850 \mathrm{~m}$ (groupements II et III) et se développe même à $1750 \mathrm{~m}$ dans le Rio Gallego et son affluent $\left(\mathrm{T}^{\circ} \max\right.$. relevée $=15^{\circ} \mathrm{C}$ le 28/07/85).

\section{Genre Habroleptoides}

Notre travail confirme les observations de Thomas (1968b) selon lesquelles $H$. berthelemyi est beaucoup plus alticole et rhéophile qu' $H$. confusa $(H$. modesta sensu auct.).

Comme remarqué précédemment (Thomas op. cit.), la cohabitation des deux espèces est rare $(5 \%$ des stations à Habroleptoides). Malgré le décalage de leur période de vol, elles se font sans doute une très forte concurrence qui limite $H$. confusa audessous de $900 \mathrm{~m}$ d'altitude. Dans les Alpes, cette dernière ne paraît pas rencontrer une telle concurrence de la part d'H. auberti car elle se montre nettement rhéophile et relativement alticole (M. Sartori 1986); elle dépasse $1300 \mathrm{~m}$ en Pologne et atteint même $1400 \mathrm{~m}$ dans les monts Tatras (Kownacki 1980).

\section{Genre Habrophlebia}

H. lauta a déjà été signalée en basse altitude, des Prépyrénées (le Volp à $280 \mathrm{~m}$ : Guillouzic 1965) et des Pyrénées-Atlantiques (le Lissuraga entre 75 et $175 \mathrm{~m}$ : Thibault 1971). Nous l'avons rencontrée en abondance dans le ruisseau lent d'Arriou Tort (groupement IIIb) et dans le ruisseau assez lent et calcaire de Serres à 820 et $830 \mathrm{~m}$ (groupement IIIa). La présence d' $H$. lauta à une telle altitude va dans le sens des observations de Landa (1957) en Tchécoslovaquie, selon lesquelles cette espèce se montre un peu plus alticole et rhéophile qu' $H$. fusca-absente en vallée d'Ossau - mais surtout plus eurytope vis-à-vis du substratum et de la vitesse du courant.

\section{Genre Ephemera}

E. danica est l'espèce européenne de ce genre la plus fréquente et, semble-t-il, la plus adaptée au fonds pierreux (Macan 1979, Verneaux 1972, Whelan 1980). Sowa (1975) l'a observée jusqu'à $900 \mathrm{~m}$ dans le massif de Babia Gora, ce qui correspond à nos propres captures en vallée d'Ossau. Cette espèce peut se montrer modérément rhéophile malgré son mode de vie fouisseur et est capable de coloniser des ruisseaux relativement froids (st. 22).

\section{Conclusion générale}

Cette étude avait pour but de préciser l'écologie de 30 espèces d'Ephéméroptères recensées dans le réseau hydrographique du Gave d'Ossau. Nos observations sont souvent différentes de celles de Puig (1980), ne serait-ce qu'en raison de l'exposition de cette vallée vers le Nord.

Deux analyses factorielles des correspondances ont mis en évidence les relations espèces-paramètres des milieux et ont permis de distinguer cinq groupements d'espèces caractéristiques de cinq biotopes lotiques montagnards : I ruisseaux très froids de haute altitude, II torrents de moyenne montagne et de piémont et ruisseaux de haute altitude à températures estivales relativement élevées, IIa rivières de piémont, IIla ruisseaux assez lents et à forte teneur en calcium et IIIb ruisseaux à faible courant et à fond vaseux.

Les limites exactes des groupements II et IIa sont encore difficiles à préciser. A l'avenir, il sera peutêtre nécessaire de scinder le groupement II en deux sous-groupes pour séparer les torrents de moyenne montagne-piémont et les ruisseaux de haute altitude à températures estivales élevées. Le peuplement en Ephéméroptères de ces deux biotopes présente cependant de grandes similitudes.

Pour mettre en évidence les préférences écologiques des espèces, nous avons étudié plus particulièrement l'influence des facteurs du milieu sur la distribution spatiale des Ephémères. Le régime thermique et la vitesse du courant apparaissent nettement comme les principaux facteurs de répartition en montagne; l'altitude, la surface du bassinversant, le numéro d'ordre du cours d'eau, le régime hydraulique et les caractéristiques physicochimiques jouent aussi un rôle important. 
Les espèces qui restent les moins bien connues sont celles qui appartiennent aux genres Ecdyonurus et Epeorus car leurs larves sont difficilement identifiables dans l'état actuel de nos connaissances. Ces espèces devront faire l'objet d'études taxonomiques ultérieures pour mieux connaître leur écologie. Des élevages seront probablement nécessaires pour obtenir du matériel à l'état imaginal, et ce, d'un grand nombre de stations.

\section{Remerciements}

C'est pour nous un plaisir de remercier M. J. Lauga (Toulouse) pour son aide apportée lors du traitement mathématique des données.

\section{Travaux cités}

Angelier (E.), Angelier (M.L.) \& Lauga (J.) 1985. Recherches sur l'éco logie des Hydracariens (Hydrachnellae, Acari) dans les eaux courantes. Annls Limnol, 21 (1) : 25-64.

Belfiore (C.) 1983. Efemerotteri (Ephemeroptera). In : Guide per it riconoscimento delle specie animali delle acque interne italiane. $n^{\circ} 24$. Verona. 113 p.

Berthélemy (C.) \& Thomas (A.) 1967. Note taxonomique sur Epeo rus torrentum Eaton, 1881 et E. assimilis Eaton, 1885 (Ephemeroptera, Heptageniidae). Annls Limnol., 3 (1): $65-74$

Bertrand (H.) \& Vertier (M.L.) 1949. Contribution à la biogéographie des Ephéméroptères des Py rénées. Bull. biol. Fr. Belg., 83 (I) : 1-24.

Brehm (V.) \& Ruttner (F.) 1926. Die Biozönosen des Lunzer Gewäs ser. Internat. Rev, ges. Aydrobiol., 16:330-392

Décamps (H.) 1967. Ecologie des Trichoptères de la vallée d'Aure (Hautes-Pyrénees). Annls Limnol, 3 (3) : 399-577.

Guillouzic (M.) 1965. Cycles de développement de quelques Ephé méroptères du Volp. Diplóme d'études supérieures. Univ. Tou louse, $34 \mathrm{p}$.

Humpesch (U.H.) \& Elliott (J.M.) 1980. Effect of temperature on the hatching time of eggs of three Rhithrogerta spp. (Ephemeroptera) from austrian streams and an english stream and river. J. Anim. Ecol, 49: 643-661.

Illies (J.) 1952. Die Mölle. Faunistisch-okologische Untersuchungen an einem Forellenbach im Lipper Bergland. Arch. Hydrobiol. $46: 424-612$.

Kamler (E.) 1965. Thermal conditions in mountain waters and their influence on the distribution of Plecoptera and Ephemeroptera larvae. Ekol. pol. (ser. A) $13\left(n^{\circ} 20\right): 377414$.

Kownacki (A.) 1980. Taxocenes of Ephemeroptera in unpolluted and polluted streams of the Tatra mountains. In : Advances in Ephemeroptera Biology, J.F. Flannagan \& K.E. Marshall eds, Plenum, New-York : pp 405-418.

Krno (I.) 1978. O vyskyte nových druhov podeniek (Ephemeroptera) na slovensku. Biologia, 33 (2) : 153-155

Landa (V.) 1957. Prispevek $k$ rozsireni systematice, vyvoji a ekolo gii druku Habrophlebia fusca (Curt.) a Habrophlebia lauta Mc Lachl. (Ephemeroptera). Cas. csl. Spol. ent., 54 (2) : 148-156.
Macan (T.T.) 1952. Taxonomy of the nymphs of the british species of Leptophlebiidae (Ephem.). Hydrobiologia, 4 (4): 363-376.

Macan (T.T.) 1957. The Ephemeroptera of a stony stream. J. Anim. Ecol., $26: 317-342$.

Macan (T.T.) 1962. Ecology of aquatic insects. A. Rev. Ent. 7 : 261.288 .

Macan (T.T.) 1979. A key to the nymphs of British Ephemeroptera. F.B.A. scient. publ. $\mathrm{n}^{\circ} 20.3^{2}$ edition. 80 p.

Maitland (P.S.) 1980. The habitats of british Ephemeroptera. In: Advances in Ephemeroptera Biology, J.F. Flannagan \& K.E. Marshall eds., Plenum, New-York: 123-139.

Puig (M.A.) 1980. Contribucio a l'estudi de l'ecologia comparada dels Plecopters i Efemeropters d'Andorra. Buthl. Inst. cat. Hist. nar., 45 (sec. Zool.), $3: 77-87$.

Sartori (M.). 1986. Révision taxonomique du genre Habroleptoides Schönemund, 1929 (Ephemeroptera, Leptophlebiidae) IHI. Description de $H$. annae nov. sp. et de $H$. thomasi nov. sp. et synthèse finale des stades ailés. Revue suisse Zool, 93 (4): $919-949$.

Sowa (R.) 1973. Taxonomie et écologie de Caenis beskidensis sp. n., des Carpates polonaises (Ephemeroptera, Caenidae). Bull. pot. Sct. (ser. Sci. biol.), 21 (5) : 351-355.

Sowa (R.) 1975. Ecology and biogeography of mayflies (Ephemeroptera) of running waters in the Polish part of the Carpathians. 1. Distribution and quantitative analysis. Acta Hydrobiol, 17 (3) : 223-297.

Sukop (I.) 1973. Annual cycle of mayflies (Ephemeroptera) in a karstic stream. Acta ent. bohemoslov. 70 (2) : 81-85.

Thibault (M.) 1971. Le développement des Epheméroptères d'un ruisseau à truites des Pyrénées-Atlantiques, le Lissuraga. Annls Limnol., 7 (1): 53-120.

Thomas (A.) 1968a. Sur la taxonomie de quelques especes d'Ecdyo rurus du Sud-Ouest de la France (Ephemeroptera). Annls Limnot. 4 (1): 51.71 .

Thomas (A.) 1968b. Habrophlebia (Habroleptoides) berthelemyi, $\mathbf{n}$. sp. des Pyrénées (Ephemeroptera, Leptophlebiidae). Annls Limnol., 4 (2) : $219-224$.

Thomas (A.) 1970. Taxonomie et répartition des Ephéméroptères et de quelques Diptères aquatiques (Tipuloidea et Psychodidae) des Pyrénées. Thèse de spécialité, Univ, Toulouse. $105 \mathrm{p}+\mathrm{XX}$ pl.

Thomas (A.G.B.) 1975. Ephéméroptères du Sud-Ouest de la France. I. Migrations d'imagos à haute altitude. Annls Limnol., 11 (1): 47-66.

Thomas (A.G.B.), Prévot (R.) \& Vinçon (G.) 1986. Deux Ephéméroptères nouveaux pour la faune de France : Baetis pentaphlebodes Ujhelyi, 1966 (Baetidae) et Caenis beskidensis Sowa, 1973 (Caenidae). Bull. Soc. Hist. nat. Toulouse, $122: 179$.

Verneaux (J.) 1972. Faune dulçaquicole de Franche-Comté. Le Bassin du Doubs (Massif du Jura). Qualrième partie : les Ephéméroptères. Anmls scient. Univ. Besançon, 3 e sér., $8: 3-14$.

Vinçon (G.). 1987. Comparaison de la faune benthique des vallées d'Aure et d'Ossau, en vue de l'élaboration d'une méthodologic de surveillance des cours d'eau de montagne. Thèse DocteurIngénieur, Univ. Toulouse, $\mathbf{n}^{\circ} 960,381 \mathrm{p}$.

Vinçon (G.). Etude hydrobiologique de la vallée d'Ossau (PyrénéesAtlantiques). II. Le milieu et la structure du peuplement. Annts Limnol., à paraitre.

Whelan (K.F.) 1980. Some aspects of the biology of Ephemera danica Müll. (Ephemeridae : Ephemeroptera) in irish waters. In : Advances in Ephemeroptera Biology, J.F. Flannagan \& K.E. Marshall eds., Plenum, New York : 187-199. 